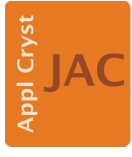

JOURNAL OF

APPLIED

CRYSTALLOGRAPHY

ISSN 1600-5767
Keywords: book reviews; small-angle X-ray and neutron scattering; static and dynamic light scattering.

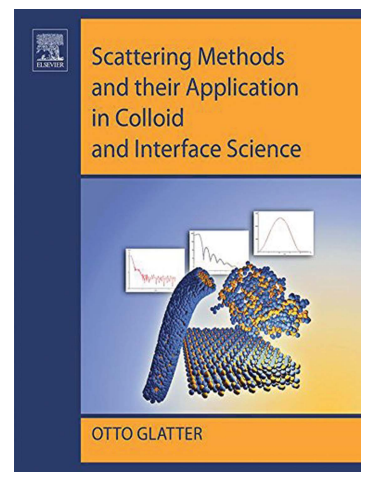

(C) 2019 International Union of Crystallography

\section{Scattering Methods and their Application in Colloid and Interface Science. By Otto Glatter. Elsevier, 2018. Paperback pp. 404. Price USD 225. Paperback (ISBN 9780128135808), ebook (ISBN 9780128135815).}

\author{
Wim Bras ${ }^{\mathrm{a} *}$ and Michal Hammel ${ }^{\mathrm{b} *}$ \\ a Oak Ridge National Laboratory, PO Box 2008, Oak Ridge, TN 3783, USA, and ${ }^{\mathbf{b}}$ Molecular Biophysics and Integrated \\ Bioimaging, Lawrence Berkeley National Laboratory, Berkeley, CA 94720, USA. *Correspondence e-mail: \\ brasw@ornl.gov, mhammel@|bl.gov
}

This book has a lot to offer. It maybe should not be seen as a comprehensive text on scattering techniques used in colloidal science but instead recognized as an overview of the long and impactful scientific career of the author, and his contributions to the development of X-ray and light scattering methods, with an emphasis on his research in colloidal systems. The fact that this is a single-author book gives it the advantage that there is a uniformity of style and no needless repetition. This lack of uniformity was one of the main drawbacks of the earlier, multi-author book edited by the author and his mentor (Glatter \& Kratky, 1982). But despite these shortcomings it served for many years, certainly in the pre-internet era, as one of the two 'bibles' of small-angle scattering, the other being the quintessential text of Guinier \& Fournet (1955).

The drawback of the present text being a single-author book, as pointed out by the author himself in the introduction, is that the subject matter is limited to his own area of expertise and omits several subject areas that fall outside his 'know-how' but which a new student of scattering techniques might want to have seen included. Personally, we do not find this a serious problem, especially as the field of small-angle scattering has expanded in the past two decades to cover so many different research areas. It is probably impossible to write a comprehensive book covering all subjects that scattering touches, and one might be better off with a single text treating a particular subject in depth. For this, the author is well positioned.

The book starts with a chapter that is filled with the fundamental heavy-duty mathematical descriptions of the interference of waves, Rayleigh-Debye-Gans formalism, and the rigorous mathematical relation between scattering and the Fourier transformation of density fluctuation, all of which are part of the basic underpinnings of the scattering techniques. When used as a depository from which one can refresh the memory regarding the exact formulation of equations this is very useful, but it might be less appealing to the large group of small-angle scattering practitioners who see the technique more as a workhorse tool and are less interested in its foundations. This group has to wait for the later chapters to whet their appetite.

Chapter 2 deals with general theorems of small-angle scattering and special cases. The derivations of commonly used relations are given. There is, however, a somewhat uneasy mixture of thoroughness and omissions. The derivations are well worked out, showing the tutorial craftsmanship that has made the author such a popular lecturer throughout the years, but the applications generally need an approximation and boundary conditions applied. What these are is often left open or has to be gleaned from figures in later chapters. Clear statements of what these boundary conditions are, and what the consequences are if the scattering relations are applied outside the range for which the derivations are valid, would have been very helpful for novices in the field. Notwithstanding this comment, it is very useful to have these derivations written out so that interested students can find that, for instance, what often is called a Guinier or Porod law is in fact an approximation.

The theoretical treatment of the inverse scattering problem for dilute systems in Chapter 3 is very instructive, with the right kinds of warnings and examples of what variations in parameters can do to scattering curves and the pair distance distribution 
functions. The same can be said for the handling of concentration and polydispersity effects in the subsequent chapter.

In Chapter 4 the author describes in a simplified and very descriptive way how small-angle X-ray or neutron scattering results on the 'absolute intensity scale' can be used to obtain fundamental sample parameters. There is something of a missed opportunity when dealing with the subject of absolute intensities, in particular the effects that the instrumentation can have in these determinations. For instance, the use of water as an absolute calibrant in X-ray scattering relies on the count-rate linearity of the detectors used. Here insights gained by the author during his extensive career would have been most welcome, particularly around the experimental details. Nevertheless, it is very useful to have all the relevant procedures for the different experimental probes written out in detail.

The chapters on contrast variation and instrumentation feel a bit unbalanced, where some subjects receive an in-depth treatment whilst other sections give the impression that they have just been added for completeness sake. This approach probably reflects the areas of expertise of the author, but a better balance could have been obtained if the areas having a less in-depth treatment had been supplemented by some references to recent literature. More attention could also have been given to the possibilities of modern synchrotron radiation sources where, beside the high flux, the high collimation and energy tunability have opened up many new techniques and research areas.

The chapter on numerical methods deals mainly with indirect Fourier transform methods, which the author pioneered and perfected. After a disappointing start, where the primary data treatment is somewhat superficially discussed, one arrives at an elaborate treatment of the underlying principles and mathematics of the methods and a set of well chosen examples that illustrate beautifully the different problems that can be solved and the pitfalls that one might encounter. Glatter's indirect Fourier transform method (supplemented later with the general indirect Fourier transform method) was the only (analysis) game in town for a long time, and the underlying ideas still form the basis of more recently developed methods. But in many laboratories, it has now been supplemented with or superseded by other techniques. It would have been beneficial if these later methods had been signposted and referenced.

In many cases, and for good reason, X-ray and neutron scattering are used as part of a bigger story in characterizing the structure and properties of materials, and as a consequence are treated as a 'black box' technique where the user inserts samples at one end and receives the analyzed data at the other end. This is even more the case for static and dynamic light scattering and, with manufacturers often not providing access to the underlying soft- and hardware, it will be difficult for the users of this kind of equipment to relate the extensive mathematical treatment the author provides to the results they can obtain from modern light scattering equipment. This raises a philosophical question: if results are obtained with non-publicly available software code and hardware settings, is the scientific method still being applied? This is not something that we would like to delve into in this forum. All we can say is that the author has provided the reader with an accessible fundament upon which the light scattering results are based and can be understood.

The later chapters are a useful tour through a variety of different scientific and experimental problems all encountered during the author's long scientific career. Here the emphasis shifts from technique oriented towards colloid research. There is a wealth of wisdom to be found in these chapters, but one should not expect to find a comprehensive overview of the applications of scattering. Even though most of the examples are somewhat dated it is interesting to take the original manuscripts and use the remarks found in this book, which give insights into the author's thinking. This is useful for everyone who would like to gain a deeper understanding about the fundamentals of the small-angle scattering methods. This is not surprising since, after all, developing scattering methodology has been one of the cornerstones of the author's long and illustrious career.

A publishing technical note is that the index is rather incomplete. One could have expected the publisher to have applied software to remedy this shortcoming. Another publisher-related issue is that the book is only available in softcover and quite highly priced. The pricing would have been acceptable for a hardcover version.

For readers who have followed the long career of the author and, on occasion, have enjoyed his seminars, many of the figures in the book will look like old friends. The drawback of this is that 30 -year-old figures do lack the graphical finesse which is so common in modern texts. A similar age-related issue is the many references to books that have been out of print for many years. Updated figures and references might not have improved the scientific content, but it can be a major factor in the appeal of the book and its place in the market. We can strongly advise potential buyers not to let this somewhat aged look influence their decision. The book offers a welcome overview of many aspects of small-angle scattering methodology and will become the first book that one grabs off the shelf to refresh one's memory with respect to theoretical issues.

\section{References}

Glatter, O. \& Kratky, O. (1982). Small Angle X-ray Scattering. London: Academic Press.

Guinier, A. \& Fournet, G. (1955). Small Angle Scattering of X-rays. New York: John Wiley \& Sons. 